INPLASY

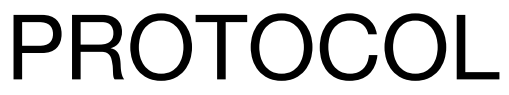

To cite: Qian et al. Effect of moxibustion combined with HAART on CD4(+) T cells in HIV/AIDS patients: a protocol for systematic review and meta-analysis. Inplasy protocol 202150041. doi:

10.37766/inplasy2021.5.0041

Received: 11 May 2021

Published: 11 May 2021

Corresponding author: Junwen Wang

wangjunwen_1963@126.com

Author Affiliation:

Hunan University of Chinese Medicine.

Support: No.2020010.

Review Stage at time of this submission: Preliminary searches.

Conflicts of interest:

None declared.

\section{Effect of moxibustion combined with HAART on CD4(+) T cells in HIV/AIDS patients: a protocol for systematic review and meta-analysis}

Review question / Objective: The aim of this review of randomized controlled trials is to evaluate the effect of moxibustion on CD4(+) T cells in HIV/AIDS patients.

Condition being studied: As the primary therapy in the treatment of HIV/AIDS, highly active antiretroviral therapy (HAART) has been routinely applied into the treatment of HIV/ AIDS, but severe toxic effects and poor immune reconstruction by an extended taking of antiviral drugs are the problems that can not be ignored. Moxibustion, as a conventional means of Chinese medicine, has been put into the treatment of HIV/AIDS in recent years due to its good therapeutic effects and high safety. The purpose of this study is to explore the effect of moxibustion on CD4+ in AIDS patients, providing evidence for its regulatory influence on immunity.

INPLASY registration number: This protocol was registered with the International Platform of Registered Systematic Review and Meta-Analysis Protocols (INPLASY) on 11 May 2021 and was last updated on 11 May 2021 (registration number INPLASY202150041).

Condition being studied: As the primary therapy in the treatment of HIV/AIDS, highly active antiretroviral therapy (HAART) has been routinely applied into the treatment of HIV/AIDS, but severe toxic effects and poor immune reconstruction by an extended taking of antiviral drugs are the problems that can not be ignored. Moxibustion, as a conventional means of Chinese medicine, has been put into the treatment of HIV/ AIDS in recent years due to its good 
therapeutic effects and high safety. The purpose of this study is to explore the effect of moxibustion on CD4+ in AIDS patients, providing evidence for its regulatory influence on immunity.

\section{METHODS}

Participant or population: The population will be composed of individuals who are over 18 years old with HIV/AIDS (as diagnosed by a clinician, or using any recognized diagnostic criteria).

Intervention: The test group will receive the therapy of moxibustion in combination with HAART. The moxibustion therapy will mainly include the mild moxibustion or other moxibustions by acting on Shenque Point, Zhongwan Point, Guanyuan Point, and other specific acupoints. Antiviral therapy will be composed of three-drug regimen containing a nonnucleoside reverse transcriptase inhibitor (NNRTI), a protease inhibitor (PI), plus one nucleoside reverse transcriptase inhibitor (NRTI).

Comparator: The control group will only accept highly active aniretroviral therapy.

Study designs to be included: The study style is randomized clinical trials (RCTs).

Eligibility criteria: Adults who are over 18 years old with HIV/AIDS (as diagnosed by a clinician, or using any recognized diagnostic criteria).

Information sources: PubMed, the Cochrane Central Register of Controlled Trials, EMBASE, China National Knowledge Infrastructure (CNKI), WanFang Database, Chinese Scientific Journal Database (VIP) and Chinese Biomedical Literature Database (CBM).

Main outcome(s): CD4(+) T cells.

Additional outcome(s): CD8(+) T cells, CD4(+)/CD8(+), T lymphocyte count, IL-2, IL-7, clinical symptom and sign scores, HIV viral load, WHOQOL-HIV and Karnovskey Score.
Quality assessment / Risk of bias analysis: Two authors will independently assess the risk of bias of each included study following the domain-based evaluation described in the Cochrane Handbook for Systematic Reviews of Interventions. This assessment tool addresses seven specific items: sequence generation, allocation concealment, blinding of participants and personnel, blinding of outcome assessment, incomplete data, selective outcome reporting, and other issues relating to bias. Each item will be evaluated at three levels: low risk, high risk and unclear.

Strategy of data synthesis: For the extracted data, a qualitative synthesis and analysis will be performed. If there are at least five studies with the same outcome, we will pool the results into Review Manager to conduct a quantitative analysis (meta-analysis) by using a random effects model, with mean differences for continuous outcomes, and calculate $95 \%$ confidence intervals and two sided $P$ values for each outcome. A 2-tailed $P<0.05$ was considered statistically significant.

Subgroup analysis: We anticipate clinical heterogeneity in the effect of the intervention and we propose to conduct, where possible, the following sub-group analyses: 1. Treatment time (e.g., < 6 months or > 6 months); 2. CD4(+) $\mathrm{T}$ cells baseline values (e.g., $<200 / \mu \mathrm{L}$ or $>200 / \mu \mathrm{L}$ ); 3. Different moxibustion methods (e.g., the mild moxibustion vs other moxibustions); 4 . TCM syndrome (e.g., spleen-kidney yang deficiency vs other syndromes).

Sensitivity analysis: To identify the quality and credibility of the results in this review, we will conduct the sensitive analysis, which is aimed at eliminating the possibility of false positives and investigating the reliability of the composite statistical results in our meta-analysis.

Language: English.

Country(ies) involved: China. 
Keywords: CD4+ T cells, HAART, HIV/AIDS, moxibustion, systematic review.

Contributions of each author:

Author 1 - Zhenzhen Qian.

Email: qianzhenzhendoctor@126.com

Author 2 - Yujin Zhang.

Email: 346281180@qq.com

Author 3 - Xiaoli Xie.

Email: 809742111@qq.com

Author 4 - Junwen Wang.

Email: wangjunwen_1963@126.com 